\title{
CARACTERÍSTICAS NEUROMOTORAS, PONDO-ESTATURAIS E BIOPSICOSSOCIAIS DE LACTENTES
}

\section{NEUROMOTOR, GROWTH AND BIOPSYCHOSOCIAL PROFILE OF LATENTS}

Joyce Ribeiro Rothstein ${ }^{1}$

Thais Silva Beltrame ${ }^{2}$

Rothstein JR e Bertrame TS. Características neuromotoras, pondo-estaturais e biopsicossociais de lactentes. Rev Bras Cresc e Desenv Hum 2011; 21(2): 269-281.

\section{RESUMO}

A pesquisa tem o objetivo de descrever as características neuromotoras, biopsicossociais e pondo-estaturais de lactentes. Esta pesquisa foi realizada no Município de Fraiburgo - SC. A amostra foi composta de 80 lactentes; sendo que o grupo experimental foi constituído de 25 lactentes com histórico de risco biológico e o grupo controle de 55 lactentes sem histórico de risco. Os instrumentos utilizados para coleta dos dados foram: Neonatal Behavioral Assessment Scale, formulário biopsicossocial e cartão de saúde da criança. Os dados foram tratados através do programa SPSS versão 13.0. Os testes estatísticos utilizados foram o teste $t$ para amostras independentes e teste U- Mann Witnney. Em relação às condições biopsicossociais, constatou-se prevalência de mães casadas, com ensino médio ou superior completo e que exerciam atividade remunerada. O tipo de parto mais freqüente foi o parto Cesário em ambos os grupos. Os lactentes do grupo de risco apresentaram índice de Apgar no primeiro e quinto minuto, perímetro cefálico, peso e comprimento ao nascimento inferior aos do grupo sem histórico de risco. Os lactentes com histórico de risco apresentaram desempenho neuromotor inferior aos lactentes sem histórico de risco em relação a organização e aos reflexos, bem como apresentaram padrão de força da mão direita e esquerda inferior ao grupo sem histórico de risco. Este estudo demonstrou que lactentes com histórico de risco apresentam características neuromotoras, biopsicossociais e pondo-estaturais diferenciadas das apresentadas por lactentes sem histórico de risco.

Palavras-chave: crescimento; desenvolvimento infantil; prematuridade; grupos de risco; lactente.

1 Fisioterapeuta, Mestra em Ciências do Movimento Humano pela Universidade do Estado de Santa Catarina - CEFID/UDESC. Centro de Ciências da Saúde e do Esporte - CEFID.

2 Educadora, Doutora em Ciências do Movimento Humano pela Universidade Federal de Santa Maria - UFSM. Centro de Ciências da Saúde e do Esporte.

Correspondência para: Joyce Ribeiro Rothstein. Rua Dr Heitor Blum, 372. Estreito Florianópolis CEP 88075110. E-mail: joycefisio@gmail.com - Telefone: 048(9914-5828). 


\begin{abstract}
The present study aimed to describe the neuromotor, biopsychosocial and weight-height characteristics of infants. This research was accomplished in Fraiburgo-SC. The sample was composed of 80 infants, the experimental group was composed of 25 infants with biological risk history and the control group was composed of 55 infants without risk history. The instruments used for the data collection were: Neonatal Behavioral Assesment Scale, biopsychosocial form and the child health card. The data analysis was accomplished by SPSS system, version 13.0. The statistic tests used were: $t$ test to independent sample and $U$ Mann Witnney test. In the biopsychosocial conditions it was found prevalence of married mothers, with complete instruction and remunerate activity. The most common type of parturition was Cesarean in either groups. The infants of the risk group presented reduced levels of Apgar index in the first and fifth minute, cephalic perimeter, weight and length at the birth compared to the control group. The infants with risk history demonstrated lower neuromotor performance compared to the control group, and also presented grip pattern of the right and the left hands lower than the control group. This study showed that infants with biological risk factors present differential neuromotor, growth and biopsychosocial profile than children without biological risk.
\end{abstract}

Key words: growth; child development; prematurity; biological risk; nurslings.

\section{INTRODUÇÃO}

O Desenvolvimento Humano é baseado em como e por que o organismo humano cresce e se modifica no decorrer da vida. Referese às mudanças que ocorrem ao longo do tempo de maneira ordenada e relativamente duradoura afetando as estruturas físicas e neurológicas, os processos de pensamento, as emoções, as formas de interação social e muitos outros comportamentos ${ }^{1,2}$.

Lactentes que vivem em países em desenvolvimento estão expostos a vários fatores de riscos, tais como a alta prevalência de doenças e gestações desfavoráveis e/ou incompletas e condições socioeconômicas e biopsicossociais adversas Tal cadeia de eventos negativos faz com que esses lactentes tenham maior chance de apresentar atrasos em seu potencial de crescimento e desenvolvimento $^{3,4}$.
Muitos são os fatores que podem trazer atraso no crescimento do feto bem como afetar de forma direta permanentemente ou temporariamente a estrutura cerebral do lactente, colocando em risco o desenrolar de suas habilidades motoras. Dentre estes fatores encontram-se: prematuridade, presença de enfermidades crônicas, desnutrição, doenças genéticas, eventos pré-natais e perinatais adversos, exposição intrauterina do feto a drogas, cigarro, álcool ou outros tipos de substancias tóxicas, hipertensão materna, transtornos cromossômicos, infecções congênitas medicamentos usados pelas mães durante a gestação, enfim, várias causas podem interferir no desenvolvimento do feto e conseqüentemente no desenvolvimento do lactente, muitas vezes indicando anormalidade no sistema nervoso central do mesmo $^{3,5}$.

Fatores como baixo peso ao nascer, prematuridade, presença de defeitos congênitos e 
asfixia neonatal estão entre as principais causas de mortalidade neonatal em várias localidades do Brasil, porém, nos últimos anos, muitos avanços tecnológicos ocorreram nas ciências da saúde, implementando as Unidades de Terapia Intensiva e dispondo de uma melhor assistência ao recém-nascido (RN) de risco $^{6,7}$. Esses avanços contribuíram não apenas para o aumento do número de lactentes sobreviventes a intercorrências gestacionais, como também na melhora de sua qualidade de vida $^{6,8}$.

Assim, o objetivo é descrever as características neuromotoras, biopsicossociais e pondo-estaturais de lactentes.

\section{MÉTODO}

Em relação às características desta pesquisa, trata-se de uma pesquisa descritiva diagnóstica do tipo transversal, baseada na comparação entre grupos com delineamento quase experimental, visto que apresenta grupo controle, porém nenhuma intervenção foi realiza junto ao grupo experimental. A presente pesquisa obteve parecer favorável do Comitê de Ética em Pesquisa em Seres Humanos (CEP) (Número de Referência 193/2007).

Esta pesquisa foi realizada no Município de Fraiburgo - SC na Unidade Central de Saúde, local que disponibiliza os serviços de Teste do Pezinho e de Vacinação. A população deste estudo foi representada por lactentes com idade de zero a dois meses de vida ${ }^{2}$, e apresentaram registro de nascimento junto as unidades de Saúde do município de Fraiburgo-SC no período de abril a setembro de 2008 .

A seleção da amostra foi realizada de forma aleatória considerando-se os seguintes critérios de inclusão: apresentar Registro de Nascimento na cidade de Fraiburgo-SC; possuir o termo de consentimento assinado pelo responsável da criança e ter idade entre zero e dois meses de vida. Após determinação da amostra, os lactentes avaliados foram separados em dois grupos (controle e experimental) e dentro destes grupos, foram subdivididos em quatro subgrupos de acordo com sua faixa etária, classificadas da seguinte forma: Grupo 1 (G1= um a 15 dias), Grupo 2 (G2=16 a 31 dias), Grupo 3 (G3= 32 a 47 dias) e Grupo $4(\mathrm{G} 4=48$ a 63 dias $)$.

O grupo experimental foi constituído de lactentes com histórico de risco biológico, ou seja, lactentes que apresentaram em seu histórico condições biológicas que colocaram o seu desenvolvimento em risco, tais como: prematuridade (PT), baixo peso (BP), crescimento intrauterino retardado, defeitos congênitos, asfixia perinatal e encefalopatia hipóxicoisquêmica, hemorragia intracraneal, displasia broncopulmonar, anormalidades bioquímicas (hipoglicemia, policitemia, hiperbilirrubinemia), infecções neonatais, crises convulsivas neonatais, exploração neurológica anormal e abusos de substâncias tóxicas por parte da mãe no período gestacional e traumas encefálicos durante o parto ${ }^{8,9}$.

O grupo controle foi composto por lactentes sem histórico de risco biológico. Os critérios para inclusão no grupo controle foram os seguintes: ter nascido a termo (entre 37 e 42 semanas), apresentar Apgar no quinto minuto superior a oito, ter peso ao nascimento adequado (igual ou maior a $2.500 \mathrm{~g}$ ) ${ }^{10}$, não apresentar alterações congênitas no Sistema Nervoso Central (SNC) (anencefalia, espinha bífida, microcefalia), alterações do estado de consciência, infecções do SNC, infecções congênitas, síndromes genéticas, os que apresentarem alterações musculoesqueléticas (paralisia braquial obstétrica, torcicolo congênito e artrogripose múltipla), hemorragia peri e intraventricular, leucomalácia periventricular ou alterações hipóxico-isquêmicas, e déficits sensoriais (auditivo-visual). Além desses foram excluídos os lactentes que apresentaram intercorrências clínicas capazes de interferir no desenvolvimento neurológico, como sepse, icte- 
rícia e ventilação mecânica por mais de sete dias ou lactentes que foram expostos a toxicomas no período gestacional.

Informações tais como Apgar no primeiro e quinto minutos, perímetro cefálico (PC), peso e comprimento ao nascimento, e intercorrências neonatais foram coletados através da verificação do Cartão de Saúde da criança. Informações relacionadas aos dados de identificação, ingestão de medicamentos, substâncias tóxicas ou doenças da mãe durante a gravidez foram informadas através de anamnese com a mãe.

Os lactentes que fizeram parte do grupo experimental e que apresentaram PT tiveram sua Idade Cronológica (IC) corrigida para termo para sua classificação no grupo de acordo com a faixa etária9.

\section{CARACTERIZAÇÃO DAAMOSTRA}

A amostra foi composta de 80 lactentes, sendo que o grupo de risco (experimental) foi composto por 25 lactentes, distribuídos da seguinte forma: $\mathrm{G} 1=8, \mathrm{G} 2=5, \mathrm{G} 3=9, \mathrm{G} 4=3 \mathrm{e}$ o grupo sem histórico de risco (controle) foi composto por 55 lactentes distribuídos da seguinte forma: $\mathrm{G} 1=15, \mathrm{G} 2=13, \mathrm{G} 3=14$, $\mathrm{G} 4=13$.

Quanto aos fatores de risco biológicos presentes no grupo experimental constataram-se os seguintes: quatro casos $(5,0 \%)$ de PT, BP e alguma intercorrência associada pós-parto, tais como necessidade de reanimação, intubação, permanência em $\mathrm{O}_{2}$, hipertensão pulmonar leve, icterícia, desconforto respiratório, infecção neonatal, anemia neonatal, seis casos $(7,5 \%)$ de PT associado a intercorrências, dois casos $(2,5 \%)$ de PT, dois casos $(2,5 \%)$ de BP, quatro casos de tabagismo materno $(5,0 \%)$, quatro casos de bossa $(5,0 \%)$ e três casos $(3,8 \%)$ de demais intercorrências (um caso de cardiopatia, um caso de má formação de traquéia e um caso de icterícia associado a incompatibilidade).

\section{INSTRUMENTOS PARA COLETA E ANÁLISE DOS DADOS}

\section{NEONATAL BEHAVIORALASSESMENT SCALE}

A Neonatal Behavioral Assesment Scale (Escala de Brazelton) é uma técnica para avaliação neonatal que permite uma avaliação específica e objetiva de diferentes características neuromotoras ${ }^{11}$.

A escala consiste de dois tipos de itens: neurocomportamentais e reflexos. O primeiro é composto de 35 variáveis que retratam aspectos sobre as ações e reações do lactente. Todos os itens são divididos em grupos de acordo com seguintes parâmetros: Habituação (mensura habilidade de se habituar a estímulos), Social Interativo ou Orientação (mensura habilidade de atender a estímulos visuais e auditivos e a qualidade de alerta apresentada durante os estímulos), Sistema Motor (mensura performance motora e qualidade do movimento e do tônus), Estado de Organização (mensura nível de excitabilidade e labilidade), Estado de Regulação (capacidade da criança de regular seu estado de consciência diante do aumento de estímulos), Sistema Autônomo (registra sinais de estresse relacionado a ajustes homeostáticos do SNC). As respostas de reflexos são avaliadas utilizando-se as respostas de 18 itens, o número de reflexos anormais é registrado ${ }^{11}$.

Todos os itens comportamentais são avaliados em uma escala de um a nove pontos (sendo nove a melhor performance), exceto oito itens da escala, que de acordo com o Manual têm seus recordes linear na pontuação cinco, seis ou oito. São os seguintes itens: Tônus (intervalo um-seis), atividade (intervalo um-cinco), pico de excitabilidade (intervalo um-cinco), o comportamento do lactente em relação ao choro diante dos estímulos (intervalo umcinco), irritabilidade (intervalo um-seis), estado de labilidade (intervalo de um-seis), susto (startles) (intervalo um-seis) e a cor da pele (intervalo um-seis). Cada ponto corresponde a 
uma definição objetiva do comportamento do lactente. Quanto maior a pontuação total da criança nos pacotes comportamentais, melhor o seu desempenho ${ }^{11}$.

Os reflexos são classificados numa escala de quatro pontos: dois se a resposta for normal, um se for fraco, três se for hipotônico, incompleto ou estereotipado, se for zero indica resposta ausente. Quanto menor a pontuação em relação aos reflexos melhor o desempenho da criança ${ }^{11}$. A cada classificação a escala atribui um valor de acordo com o tipo de reflexo, nos casos dos reflexos anormais como nistagmo e clônus o item zero tem pontuação zero, visto que é ideal que os lactentes não apresentem estes reflexos. Já para os reflexos típicos, o item dois também tem pontuação zero, os demais itens, pontuação diferenciada de zero; por isso, a pontuação que representa o melhor desempenho em relação aos reflexos é a pontuação zero.

Estados de consciência foram observados para que os itens comportamentais pudessem ser administrados e pontuados. Os estados de consciência específicos são: sono profundo (estado um), sono ativo (estado dois), sonolência (estado três), alerta ativo (estado quatro), considerável atividade motora (estado cinco) e choro (estado seis). A avaliação motora foi realizada sempre pelo mesmo pesquisador em dois ambientes: na casa do lactente ou na Unidade Central do posto de saúde na sala de pesagem dos lactentes.

\section{CARTÃO DE SAÚDE DA CRIANÇA}

Informações sobre antecedentes clínicos foram coletadas a partir do Cartão de Saúde da Criança proposto pelo Ministério da Saúde. O Cartão da Criança contém informações referentes ao estado da criança desde o nascimento: peso, comprimento, perímetro cefálico (PC), Apgar, dentre outras informações relevantes, assim como informações de intercorrências. Essas informações foram registradas junto ao Formulário Biopsicossocial.

\section{FORMULÁRIO BIOPSICOSSOCIAL}

O Formulário Biopsicossocial foi utilizado com o objetivo de registrar informações referentes aos dados de cada lactente avaliado, abrangendo informações pessoais, gestacionais, do nascimento e pós-natais, além de informações sobre o estado de saúde do lactente no dia da realização da avaliação, horário que acordou e da última mamada, peso e estatura, estado de alerta, dentre outros fatores relevantes. Informações como idade materna durante o parto, amamentação, ingestão de substâncias tóxicas durante a gestação e outras informações não encontradas no Cartão de Saúde da criança foram obtidas por meio de questionamentos à mãe do lactente.

Os resultados foram analisados através do programa Statistical Package for Social Sciencies for Personal Computer (SPSS-PC), versão 13.0. Dados referentes às condições biopsicossociais, padrão pondo-estatural e características neuromotoras foram apresentados por estatística descritiva. Na parte inferencial, utilizou-se o teste de shapiro-wilk com vista à análise da distribuição da amostra. Posteriormente, a comparação entre características neuromotoras, biopsicossociais e pondo estaturais de lactentes de risco biológico com as características de lactentes sem histórico de risco biológico foram realizadas pelo teste $U$ de Mann-Whitney ou teste t para dados independentes quando apresentaram distribuição normal. O nível de significância estabelecido foi de $5 \%(a ́=0,05)$.

\section{RESULTADOS E DISCUSSÃO}

\section{CARACTERÍSTICAS NEUROMOTORAS DOS GRUPOS}

Em relação às características neuromotoras dos grupos, a Tabela 1 apresenta os valores médios dos grupos (controle e experimental) e a diferença entre as médias apresentadas.

Pode-se observar na tabela 1 que no G1, quanto aos reflexos, o grupo de risco biológi- 
Tabela 1: Comparação das Características Neuromotoras dos Grupos de risco com as características do grupo sem histórico de risco biológico de acordo com a faixa etária

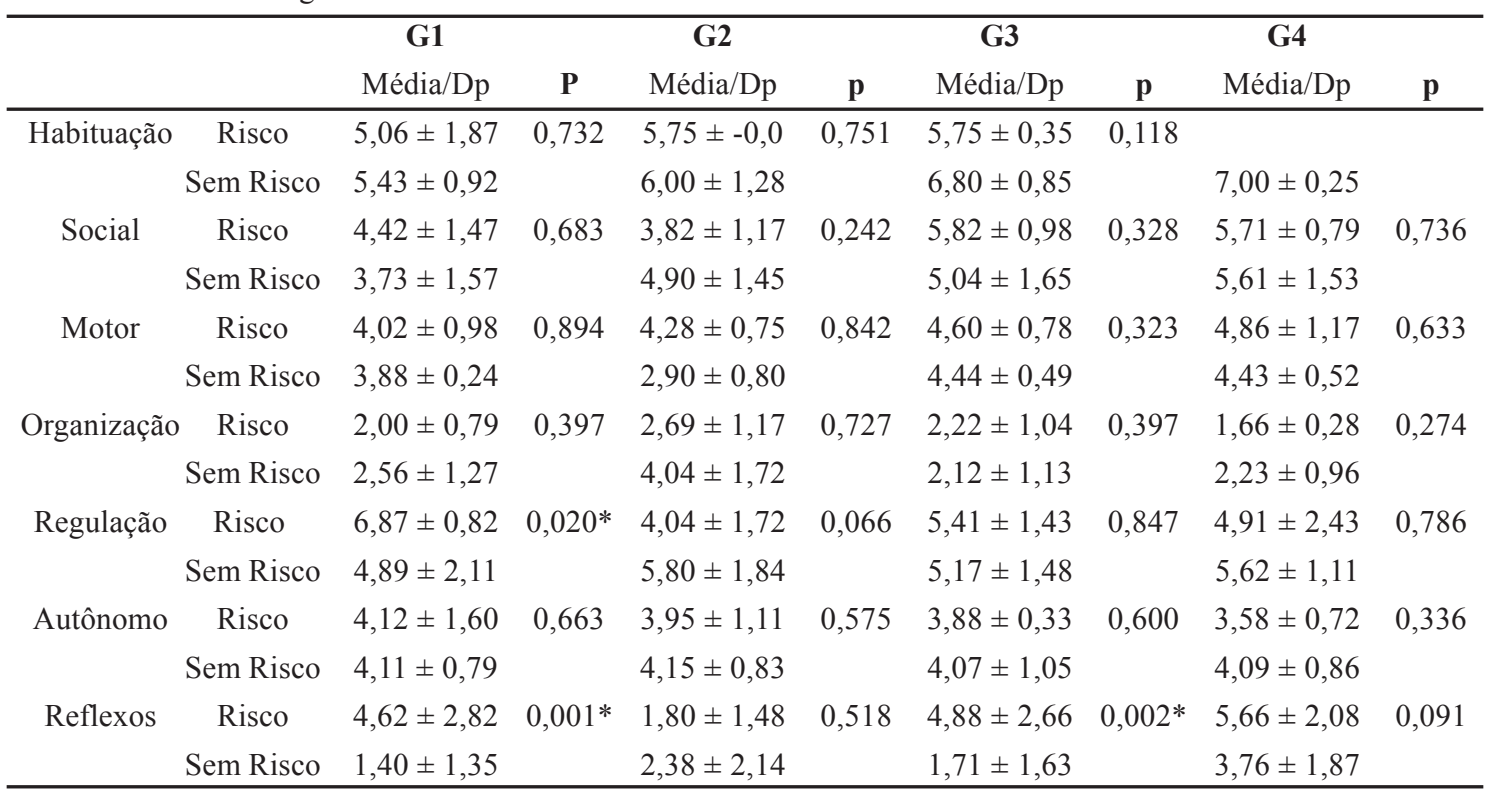

* significativo para $\mathrm{p}<0,05$; teste $t$ para dados independentes

co apresentou desempenho inferior ao grupo sem histórico de risco, visto que apresentou ausência ou presença exacerbada de reflexos apresentando média de 4,62. No G2, o desempenho do grupo de risco foi inferior em relação à habituação, à socialização/orientação, à motricidade e ao sistema autônomo. No G3 o grupo de risco apresentou desempenho inferior ao grupo sem histórico de risco quanto à habituação, ao sistema autônomo e em relação aos reflexos. No G4, em relação à organização, regulação, sistema autônomo e reflexos o grupo de risco apresentou desempenho inferior ao grupo sem histórico de risco.

Ao comparar as características neuromotoras de lactentes de risco biológico às de lactentes sem risco biológico, observa-se na Tabela 01 que foram encontradas diferenças estatisticamente significantes entre os grupos na faixa etária $G 1$, sendo que o grupo sem histórico de risco biológico apresentou média inferior, e a diferença entre esta e a do grupo com histórico de risco biológico foi significativa em relação à regulação $(p=0,020)$ e aos reflexos $(p=0,001)$. Na faixa etária $G 3$, em relação aos reflexos, a diferença encontrada entre os grupos também foi estatisticamente significante $(p=0,002)$, apresentando o grupo com histórico de risco biológico média superior em relação aos reflexos, demonstrando, portanto, desempenho inferior quando comparado ao grupo sem histórico de risco biológico (Tabela 1).

\section{CONDIÇÕES BIOPSICOSSOCIAIS}

Em relação ao estado civil houve prevalência de mães casadas e vivendo em união estável em ambos os grupos. Quanto à idade materna durante o parto, no grupo de risco as maiores proporções encontradas foram nas faixas etárias entre 19 e 23 anos $(n=9,36 \%)$ e entre 34 e 38 anos ( $n=6,24 \%)$; e no grupo sem histórico de risco entre as idades de 24 e 28 anos $(n=15,27,27 \%), 29$ e 33 anos, e 34 e 38 anos com a mesma proporção $(n=13,23,64 \%)$, respectivamente.

Pode-se observar neste estudo que, apesar da média de idade durante o parto de toda a 
amostra ter sido de 28 anos, o número de primigestas foi maior que o número de multíparas.

Quanto ao grau de escolaridade materno, em ambos os grupos, as maiores proporções em relação à escolaridade foram relacionadas a ensino superior completo representado em $40 \%(n=10)$ no grupo de mães de lactentes com histórico de risco, e em 33\% ( $\mathrm{n}=18,15)$ no grupo de mães sem histórico de risco biológico.

Número relevante de mães que exerciam atividade remunerada foi encontrado em ambos os grupos: grupo de risco $=56 \%, \mathrm{n}=14 \mathrm{e}$ sem histórico de risco $=67,30 \%, \mathrm{n}=37$.

Em relação aos dados gestacionais, a anemia e diabetes apresentaram prevalência no grupo com histórico de risco biológico. Casos de hipertensão materna (risco $=16 \%$, $\mathrm{n}=4$; sem risco $=14,5 \%, \mathrm{n}=8$ ), hipotensão (ris$\mathrm{co}=28 \%, \mathrm{n}=7$; sem risco; $1,8 \% \mathrm{n}=1$ ), hemorragia, sífilis e edema também foram constatados. Quanto à ingestão de substâncias tóxicas na gestação, seis mães do grupo com histórico de risco $(7,5 \%)$ relataram ter fumado durante a gestação. $\mathrm{O}$ uso de outras substancias tóxicas (álcool, drogas) não foi relatado por nenhuma mãe.

Em relação aos dados de nascimento, o tipo de parto mais encontrado neste estudo foi o parto cesáreo em ambos os grupos (ris$\mathrm{co}=72 \%$, sem risco $=58,2 \%$ ). No grupo com histórico de risco observou-se a presença de parto induzido $(1,3 \%, \mathrm{n}=1)$ e fórceps $(1,3 \%, \mathrm{n}=1)$.

Com referência aos dados pós-natais a Idade Gestacional (IG) no presente estudo teve como valor médio, $37,92( \pm 1,78)$ semanas, valor mínimo de 32 semanas e máximo de 41 semanas. Nasceram com IG inferior a 37 semanas, 12 lactentes (15\%), enquanto os demais nasceram a termo (IG entre 37 e 42 semanas).

Quanto aos índices de Apgar o valor médio no primeiro minuto, no grupo de risco biológico, foi de $7,00( \pm 2,3)$ e no quinto minuto foi de $8,6( \pm 0,86)$. No grupo sem histórico de risco biológico, o valor médio no primeiro minuto foi de $7,9( \pm 1,26)$ e no quinto minuto de $9,00( \pm 0,43)$. Apenas um lactente $(1,3 \%)$ apresentou valor inferior a oito no quinto minuto. Comparando-se as médias de Apgar entre o grupo de risco e o grupo sem risco, esta foi maior no grupo sem risco tanto no primeiro quanto no quinto minuto, porém esta diferença foi estatisticamente significante somente em relação ao Apgar no quinto minuto $(\mathrm{p}=0,010)$.

\section{CARACTERÍSTICAS PONDO- ESTATURAIS DE LACTENTES DO NASCIMENTO AOS DOIS MESES DE VIDA}

Quanto às diferenças entre as características pondo-estaturais ao nascimento entre o grupo de risco e sem risco, contatou-se que a média de peso ao nascimento do grupo de risco biológico $(2,783 \pm 0,55680 \mathrm{~kg})$ foi inferior, à média apresentada pelo grupo sem histórico de risco biológico $(3,155 \pm 0,42260 \mathrm{~kg})$, sendo que esta diferença mostrou-se estatisticamente significante $(t=-3,29, p=0,001)$. E ao considerar o peso ao nascimento, oito lactentes do grupo de risco (32\%) apresentaram valores inferiores a $2.500 \mathrm{~g}$.

Em relação ao comprimento, o valor médio de comprimento ao nascimento do grupo com histórico de risco foi de $46,04 \pm 2,56 \mathrm{~cm}$ e do grupo sem histórico de risco foi de 47,51 $\pm 1,64 \mathrm{~cm}$, sendo que a diferença entre os grupos foi estatisticamente significante $(\mathrm{t}=-3,086$, $\mathrm{p}=0,003)$. O PC variou de 32 a $38 \mathrm{~cm}$ em ambos os grupos $( \pm 1,12)$ com uma média de $34,00 \mathrm{~cm}$.

Em relação às características neuromotoras dos grupos, pode-se observar que os lactentes com histórico de risco biológico apresentaram desenvolvimento e função motora diferenciados de lactentes a termo. Este fato converge com o estudo de Pedersen et al. ${ }^{12}$. Os autores verificaram que lactentes com históri- 
co de PT e BP apresentam motricidade diferenciada com relação a lactentes sem histórico de risco. O mesmo foi constatado neste estudo, visto que, tanto no G1 quanto no G3 houve diferença significativa em relação ao comportamento motor reflexo de lactentes de risco biológico em comparação a lactentes sem histórico de risco biológico.

O estudo de Diego et al. ${ }^{13}$ comparou o desempenho neuromotor dos lactentes de 80 mães com sintomas de depressão, com lactentes filhos de mães sem histórico de depressão. Os autores constataram que os filhos de mães com depressão apresentaram valores inferiores em relação ao pacote de orientação e motricidade, variáveis diferentes das que apresentaram repercussões dos fatores de risco biológicos no presente estudo.

Mães com depressão apresentam um perfil elevado de cortisol e noripinefrina prénatal, assim como níveis mais baixos de dopamina, serotonina e alterações bioquímicas tornando os lactentes destas mais propensos a nascerem prematuros e de BP. Fiedl et al ${ }^{14} \mathrm{e}$ Field et al. ${ }^{15}$ avaliaram os lactentes de 98 mães com depressão e constataram maior incidência de BP fetal, menor comprimento de fêmur e menor circunferência abdominal nos referidos lactentes ${ }^{16}$.

Quanto às condições biopsicossociais, a média da idade das mães da amostra deste estudo foi de 28 anos, dado que se assemelha ao encontrado em demais estudos ${ }^{16}$, que constataram médias de idades maternas durante o parto de 28 anos e quatro meses. No presente estudo, um número relevante de mães apresentou idade ao parto superior a 30 anos ou inferior a 25 anos. A idade materna inferior aos 20 anos segundo evidências da literatura ${ }^{17,18,19}$, traz risco tanto para mãe quanto para o lactente, aumentando a chance de BP ao nascimento. Associação entre idade materna igual ou superior a 35 anos, presente em proporções significativas neste estudo, está associada a resultados perinatais adversos $\mathrm{s}^{20,21}$.
Assim como na presente pesquisa o número de mães primigestas, também foi prevalente no estudo de Olhweiler et al. ${ }^{22}$. Estes dados refletem a menção de Azenha et al. ${ }^{17}$ de que a proporção de primíparas aumentou de $35 \%$ para $40,7 \%$ nos últimos tempos, enquanto que o número de multíparas reduziu-se à metade.

No caso deste estudo, a maioria das mães apresentou grau de instrução entre ensino médio e superior. No estudo de Fenster et $\mathrm{al}^{23}$ apenas $19 \%$ das mães tinham ensino médio completo, sendo que este número foi ainda inferior no estudo de Soares et $\mathrm{al}^{24}$, no qual somente $14 \%$ das mães chegaram a concluir as oito séries do primeiro grau escolar.

Mais de $40 \%$ das mães tinham ensino médio ou superior completo em ambos os grupos (risco e sem risco). Um maior nível de escolaridade das mães associa-se com maior variedade de estimulação e interação com lactentes, o que pode interferir no favorecimento do desenvolvimento neuromotor do lactente ${ }^{23,25}$.

Em relação às características biopsicossocias, tais como grau de escolaridade materna, atividade remunerada, tipo de parto e estado civil, não se constatou diferença estatisticamente significante entre os grupos de lactentes de risco biológico e sem risco biológico ( $>00,05$, á=0,05).

Em ambos os grupos, a maioria das mães exercia atividade remunerada. Segundo Andrade et $\mathrm{al}^{26}$ existe relação entre interação materna de maior qualidade e melhor indicador de desenvolvimento cognitivo entre $17 \mathrm{e}$ 42 meses para os lactentes cujas mães trabalhavam fora de casa.

No presente estudo casos de anemia estiveram presentes no grupo de risco biológico. Pesquisas evidenciam que a anemia na gestação relaciona-se com pobre resultado gestacional, com maior risco de PT, de BP, mortalidade perinatal e maior concentração de hemoglobina no RN, bem como aumento da mortalidade materna ${ }^{27,28}$. 
Um número relevante de mães apresentou quadro de hipertensão no período gestacional. No entanto, quando a hipertensão gestacional não cursa com complicações mais sérias, esta é inofensiva em relação do desenvolvimento neuromotor de lactentes, ou seja, a hipertensão gestacional parece não ser suficiente para causar danos neurológicos importantes ao lactente, a não ser que os níveis pressóricos elevados sejam suficientes para provocar tais danos vasculares ${ }^{29}$.

Quanto à ingestão de substâncias tóxicas durante a gestação as proporções de mães fumantes encontradas nos estudos de Fenster et $\mathrm{al}^{23} \mathrm{e}$ Young et $\mathrm{al}^{30}$ foram menores do que as observadas neste estudo. Estudos evidenciam que a nicotina é um neurotóxico para o desenvolvimento do SNC aumentando a pressão arterial do feto e diminuindo a saturação de oxiemoglobina e $\mathrm{pH}$ arterial, devido a este fato lactentes de mães fumantes tem riscos maior de nascer PT ou apresentar morte perinatal ${ }^{31}$.

O tabagismo materno tem diminuído de $26,9 \%$ para $18,5 \%$ nos últimos anos ${ }^{17}$, porém, assim como no presente estudo, no estudo de Smith et al ${ }^{32}$ o tabagismo foi o mais comum da via de administração (81\%) de substâncias tóxicas.

Dados de nascimento indicam que nesta pesquisa houve prevalência de parto cesariano evidenciando tendência encontrada na literatura $^{21,33,34}$. Segundo Guimarães e Meléndez ${ }^{19}$ maiores proporções de parto cesáreo se encontram em grupos de mães com grau de instrução superior e em mulheres com idade superior a 35 anos, perfil este, bastante característico da amostra do presente estudo. Dado bastante diferenciado foi encontrado no estudo de Pedroso e Rotta ${ }^{35}$, no qual nasceram de parto vaginal $84(79,2 \%)$ de 106 lactentes avaliadas e $20,8 \%$ nasceram de parto cesariano.

Quanto aos dados pós-natais, proporções de PT bem diferenciadas foram encontradas no estudo de Resegue et $\mathrm{a}^{36}$, no qual de um total de 211 lactentes, 104 (49,3\%) eram nascidos PT.
No período de junho de 2004 a maio de 2005 , nasceram vivos 17.219 lactentes em oito unidades de saúde, destes 3\% $(n=579)$ eram PT com IG entre 23 e $33^{37}$. Olhweiler et al ${ }^{22}$ constataram em seu estudo que $25 \%$ dos PT apresentavam IG abaixo de 30 semanas, $48 \%$ se situaram entre 30 e 34 semanas, e $27 \%$ tinham acima de 34 semanas de IG. Já no estudo de Fraga et $\mathrm{a}^{25} \mathrm{a}$ mediana da IG das lactentes da amostra foi de 31 semanas (29 a 33) inferior ao encontrado em outros estudos ${ }^{30,31}$ nos quais a IG teve média de 39 e 38,9 semanas respectivamente. A média gestacional encontrada neste estudo foi inferior a dos referidos autores.

Os índices de Apgar encontrados neste estudo apresentaram média superior a sete tanto no primeiro quanto no quinto minuto, em ambos os grupos. Dado semelhante foi encontrado no estudo de Olhweiler et al. ${ }^{22}$ em que 40 lactentes prematuros participantes do estudo, $32,5 \%$ tiveram Apgar entre oito e nove no quinto minuto, $5 \%$ apresentaram Apgar sete e $10 \%$ ficaram com Apgar dez.

Dados semelhantes também foram encontrados em outros estudos. Através de análises baseadas em uma amostra de 381 lactentes constataram-se escores de Apgar no primeiro minuto com média de $8,9^{30}$. No estudo de Manacero e Nunes ${ }^{38}$, os índices de Apgar encontrados no primeiro minuto apresentam média com valor acima de sete e nos quinto minuto a média foi superior a 8,5 .

$\mathrm{O}$ escore de Apgar entre zero e seis no quinto minuto está fortemente associado ao óbito neonatal precoce, segundo Almeida et $\mathrm{al}^{39}$. Segundo o autor, estudos constataram que o risco neonatal foi 59 vezes maior quando o Apgar no quinto minuto foi de zero a três e 13 vezes maior para valores de quatro a seis usando como padrões de referência escores de sete a 10. O autor também relata que o Apgar de primeiro minuto isolado não se mostra útil para predizer o risco de morte.

Lactentes com valores de Apgar de risco têm maior chance de apresentar alterações 
ao exame neurológico, segundo Fraga et al ${ }^{25} \mathrm{e}$ Dias et $\mathrm{al}^{40}$, que também mencionam que há uma tênue relação entre asfixia fetal e baixos índices de Apgar.

Em relação às características pondoestaturais dos lactentes o peso médio ao nascimento encontrado neste estudo foi de $3.039 \mathrm{~g}$, valor este um pouco inferior ao encontrado por Young et $\mathrm{al}^{30}$, em que o valor médio do peso de nascimento dos lactentes de seu estudo foi de $3.500 \mathrm{~g}$. Segundo Kassar et $\mathrm{al}^{41}$, lactentes que nascem com peso menor que $2.500 \mathrm{~g}$ (baixo peso ao nascer - BPN) e com peso situado entre $2.500 \mathrm{~g}$ e $2.999 \mathrm{~g}$ (peso insuficiente) estão sob alto risco de adoecer e morrer. Este fato foi apresentado por oito lactentes do grupo de risco no presente estudo.

Ao avaliar o peso de lactentes prematuros, entre 23 e 33 semanas de IG, Almeida et al ${ }^{37}$ constataram valores que variavam de 400 a $1.500 \mathrm{~g}$; essa faixa de valores encontra-se bastante inferior aos obtidos no presente estudo. No estudo de Manacero e Nunes ${ }^{38}$, no qual o autor avaliou $44 \mathrm{RN}$ prematuros, destes 14 com peso inferior a $1.750 \mathrm{~g}$ e $30 \mathrm{com}$ peso maior ou igual a $1.750 \mathrm{~g}$. Fraga et al ${ }^{25}$ verificaram que a média de peso ao nascimento de lactentes prematuros foi de 990 gramas (7101425). Na pesquisa de Almeida et $\mathrm{al}^{35}, 55 \%$ dos lactentes apresentaram peso $<1.000 \mathrm{~g}$. Olhweiler et al. ${ }^{22}$ constataram $50,5 \% \mathrm{RN}$ com peso $1.000 \mathrm{~g}$ e $1.500 \mathrm{~g}, 20 \%$ com peso inferior a $1.000 \mathrm{~g}$ e $29,5 \%$ com peso acima de $1.500 \mathrm{~g}$.

Em relação ao comprimento dos lactentes ao nascimento, Goulart et al ${ }^{34}$ relatam que, em sua pesquisa, o comprimento variou de $44 \mathrm{~cm}$ a $54 \mathrm{~cm}( \pm 1,8)$ tendo sua média em $49,49 \mathrm{~cm}$, valor semelhante ao encontrado neste estudo, visto que a média de comprimento ao nascimento foi de $47 \mathrm{~cm}( \pm 2,8)$.

$\mathrm{O}$ crescimento do feto está intimamente ligado à oferta de oxigênio e nutrientes e é influenciado por fatores que atuam em conjunto tais como fatores genéticos e ambientais ${ }^{42}$. São atribuídos a fatores genéticos $38 \%$ da variação do peso de nascimento e os $62 \%$ restantes a fatores não genéticos. Dos fatores não genéticos, metade depende de variáveis maternas e a outra metade, de causas desconhecidas. Os fatores maternos que mais influenciam o crescimen-to fetal são: paridade, nível socioeconômico, raça, altura, fumo, estado nutricional, fatores relacionados à unidade útero-placentária e hormônios. A própria nutrição materna periconceptual influencia a qualidade do crescimento fetal. A medida de comprimento reflete o potencial do crescimento e sofre menor influência diante de uma nutrição fetal inadequada, bem como não se altera com o estado de hidratação da criança. Espera-se do lactente o crescimento de um centímetro por semana conforme as curvas de crescimento intrauterino ${ }^{40}$.

Quando avaliados os valores de PC, pode-se observar que o grupo de risco apresentou valor médio de PC inferior $(33,88, \pm 1,63 \mathrm{~cm})$ quando comparado ao valor médio do grupo sem histórico de risco $(34,77, \pm 2,33 \mathrm{~cm})$. Esta diferença apresentou-se estatisticamente significante $(\mathrm{t}=-1,887$, $\mathrm{p}=0,063$ ).

O PC apresenta relação direta com o tamanho encefálico e é a medida mais poupada em casos de desnutrição. $\mathrm{O}$ aumento proporcional do PC indica crescimento adequado e melhor prognóstico neurológico. Espera-se que o crescimento do PC seja em média de um centímetro por semana ${ }^{31,35}$.

Portanto, o presente estudo constatou que, em relação às características neuromotoras, o grupo de risco biológico apresentou características diferenciadas ao grupo sem histórico de risco em relação à regulação e aos reflexos. As características biopsicossociais dos grupos são bastante semelhantes, sendo que em ambos os grupos foram contatadas: prevalência de mães casadas, mães com ensino médio ou superior concluído e com idade média durante o parto superior a 30 anos. Alterações pressóricas e nos níveis de ferro também foram constatadas em ambos os grupos. 
Em relação aos critérios pondoestaturais, o grupo de risco biológico apresentou características diferenciadas, por apresentar comprimento, perímetro cefálico e peso ao nascimento inferiores aos encontrados no grupo sem histórico de risco.

Isto reforça a necessidade da existência programas de avaliação e intervenção específicos ao cuidado do desenvolvimento de lactentes com histórico de risco. Estes programas devem contar com a atuação de profissionais de diversas áreas da saúde e da educação com o intuito de proporcionar aos lactentes o monitoramente frequente de seu desenvolvimento; especialmente até o segundo ano de

\section{REFERÊNCIAS}

1. Bronfenbrenner, U. A ecologia do desenvolvimento humano: experimentos naturais e planejados. Porto Alegre: Artes Médicas; 1996.

2. Newcombe N. Desenvolvimento Infantil: abordagem de Mussen. Porto Alegre: Artmed, 1999. p.:25-32.

3. Halpern R, Figueiras ACM. Influências ambientais na saúde da criança. Jornal de Pediatria. 2004; 80(2): 1-11.

4. Hallal CZ, Marques NR, Braccialli LMP. Aquisição de habilidades funcionais na área de mobilidade em crianças atendidas em um programa de estimulação precoce. Rev. bras. crescimento desenvolv. hum., Abr 2008, vol.18, no.1, p.27-34. ISSN 0104-1282

5. Vargas LE, Reguera CF, Losada JP, Sanz AL, Gallinato MR, Meneses AG. Deficiências: Medidas preventivas durante El período neonatal. Vox Pediátrica. 1999; 7 (1): 81-90.

6. Barbosa VC; Formiga CKMR; Linhares MBM. Avaliação das variáveis clínicas e neurocomportamentais de recém-nascidos pré-termo. Rev. bras. fisioter. 2007; 11(4): 275-281. vida. Por meio destas iniciativas, é possível que os profissionais realizem diagnósticos precoces e avaliações, a partir dos resultados obtidos, e estabeleçam metas de intervenção buscando a prevenção, a minimização e o tratamento específico da necessidade de cada criança com histórico de risco. Estas intervenções visam diminuir de forma significativa as repercussões que os déficits apresentados nas idades mais tenras possam acarretar em idades subsequentes.

Assim, os fatores de risco biológicos repercutem de forma negativa tanto no desenvolvimento motor quanto em aspectos relacionados ao crescimento e desenvolvimento do lactente.

7. Paulucci RS, Nascimento LFC. Mortalidade neonatal em Taubaté: um estudo caso-controle. Rev. Paul. Pediatr. 2007; 25(4): 358-363.

8. Magalhães LC, Catarina PW, Barbosa VM, Mancini MC, Paixão ML. Estudo comparativo sobre o desempenho perceptual e motor na idade escolar em crianças nascidas pré-termo e a termo. Arq. NeuroPsiquiatr. 2003; 61(2): 290-297.

9. Rugolo LM. Crescimento e desenvolvimento a longo prazo do prematuro extremo. J Pediatr. 2005; 81(5): 101-10.

10. Falcão MC. Avaliação nutricional do recémnascido. Pediatria. 2000; 22(3): 234-239.

11. Brazelton TB, Nuget JK. Neonatal Behavioral Assessment Scale. London: MacKeith Press; 1995.

12. Pedersen SJ, Sommerfelt K, Markestad J. Early motor development of premature infants with birthweight less than 2000 grams. Acta Paediatric. 2000; 89(6): 1456-1461.

13. Diego M, Field T, Reif MH. Prepartum, postpartum and chronic depression effects on neonatal behavior. Infant Behavior \& Development. 2005; 28(2): 155-164.

14. Field T, Diego M, Dieter J, Reif MH, Schanberg S, Kuhn C, Yando R, Bendell D. Prenatal depression effects on the 
fetus and the newborn. Infant Behavior \& Development. 2004; 27 (2): 216-229.

15. Field T, Reif MH, Diego M. Newborns of depressed mothers who received moderate versus light pressure massage during pregnancy. Infant Behavior \& Development. 2006; 29(1):54-58.

16. Field T, Miguel D, Reif MH. Prenatal dysthymia versus major depression effects on the neonate. Infant Behavior \& Development. 2008; 31(2):190-193.

17. Azenha VM, Mattar MA, Cardoso VC, Barbieri MA, Del CLA, Bettiol H. Peso insuficiente ao nascer: estudo de fatores associados em duas coortes de recém-nascidos em Ribeirão Preto, São Paulo. Rev. paul. pediatr. 2008; 26(1): 27-35.

18. Suzuki CM, Ceccon MEJ, Falcão MC, Vaz CFA. Análise comparativa da freqüência de prematuridade e baixo peso entre filhos de mães adolescentes e adultas. Rev. bras. crescimento desenvolv. hum., Dez 2007, vol.17, no.3, p.95-103. ISSN 0104-1282

19. Chalem E, Mitsuhiro SS, Ferri CP, Barros MCM, Guinsburg R, Laranjeira R. Gravidez na adolescência: perfil sociodemográfico e comportamental de uma população da periferia de São Paulo, Brasil. Cad. Saúde Pública. 2007; 23 (1): 177-186.

20. Senesi LG, Tristão EG, Andrade RP, Krajden ML, Oliveira JFC, Nascimento DJ. Morbidade e Mortalidade Neonatais Relacionadas à Idade Materna Igual ou Superior a 35 Anos, segundo a Paridade. RBGO. 2004; 26(6): 477-482.

21. Guimarães EAA, Melendez GV. Determinantes do baixo peso ao nascer a partir do Sistema de Informação sobre Nascidos Vivos em Itaúna, Minas Gerais. Rev. Bras. Saude Mater. Infant. 2002; 2(3): 283-290.

22. Olhweiler L, Silva AR, Rotta NT. Estudo dos reflexos primitivos em pacientes recém-nascidos pré-termo normais no primeiro ano de vida. Arq. Neuro-Psiquiatr. 2005; 63(2): 294-297.
23. Fenster L, Eskenazi B, Anderson M, Bradman A, Hubbard A, Barr DB. In utero exposure to DDT and performance on the Brazelton neonatal behavioral assessment scale. NeuroToxicology. 2007;28:471-477.

24. Soares NT, Guimarães ARP, Sampaio HAC, Almeida PC, Coelho R R. Padrão alimentar de lactentes residentes em áreas periféricas de Fortaleza. Rev. Nutr. 2000; 13(3): 167-176.

25. Fraga DA, Linhares MBM, Carvalho AEV, Martinez FE. Desenvolvimento de bebês prematuros relacionado a variáveis neonatais e maternas. Psicol. Estud. 2008; 13(2): 335-344.

26. Andrade PC, Linhares JJ, Martinelli S, Antonini M, Lippi UG, Baracat FF. Resultados perinatais em grávidas com mais de 35 anos: estudo controlado. Rev. Bras. Ginecol. 2004; 26(9): 697-701.

27. Sato APS, Fujimori E, Szarfarc SC, Sato JR, Bonadio IC. Prevalence of anemia in pregnant and iron fortification of flours. Texto contexto - enferm. 2008; 17(3): 474-481.

28. Engmann C, Adanu R, Lu T.-S, Bose C, Lozoff $\mathrm{B}$. Anemia and iron deficiency in pregnant Ghanaian women from urban areas. International Journal of Gynecology and Obstetrics. 2008; 101(1): 62-66.

29. Silveira RC, Procianoy RS. Lesões isquêmicas cerebrais no recém-nascido pré-termo de muito baixo peso. J. Pediatr. 2005; 81(1):23-32.

30. Young JG, Eskenazi B, Gladstone EA, Bradman A, Pedersen L, Johnson C, Barr DB, Furlong CE, Holland NT. Association Between In Utero Organophosphate Pesticide Exposure and Abnormal Reflexes in Neonates. NeuroToxicology. 2005;26 (2): 199-209.

31. Possato M, Parada CMGL, Tonete VLP. Representação de gestantes tabagistas sobre o uso do cigarro: estudo realizado em hospital do interior paulista. Rev. esc. enferm. 2007; 41(3):434-440. 
32. Smith L.M, LaGasse LL, Derauf C, Grant P, Shah R, Arria A, Huestis M, et al. Prenatal methamphetamine use and neonatal neurobehavioral outcome. Neurotoxicology and Teratology. 2008; 30(1):20-28.

33. Vries N, Erwich J, Bos A. General movements in the first fourteen days of life in extremely low birth weight (ELBW) infants. Early Human Development. 2008; 84 (11):763-768.

34. Goulart AP, Valle CF, Dal-Pizzol F, Cancelier ACL. Fatores de risco para o desenvolvimento de sepse neonatal precoce em hospital da rede pública do Brasil. Rev. bras. ter. intensiva. 2006; 18(2): 148-153.

35. Pedroso FS, Rotta NT. Neurological examination in the healthy term newborn. Arq. Neuro-Psiquiatr. 2003; 61(4):902-905.

36. Resegue R, Puccini R, Silva E. Risk factors associated with developmental abnormalities among high-risk children attended at a multidisciplinary clinic. São Paulo Med. 2008; 126 (1): 4-10.

37. Almeida MFB, Guinsburg R, Martinez FE, Procianoy RS, Leone CR, Marba STM, et al. Fatores perinatais associados ao óbito precoce em prematuros nascidos nos centros da Rede Brasileira de Pesquisas Neonatais. J. Pediatr. 2008; 84(4): 300-307.

38. Manacero S, Nunes ML. Avaliação do desempenho motor de prematuros nos primeiros meses de vida na Escala Motora Infantil de Alberta (AIMS). J. Pediatr. 2008; 84(1): 53-59.

39. Almeida MFB. Quando devemos iniciar a fototerapia em recém-nascidos pré-termo? Jornal de Pediatria. 2004; 80 (4): 256-258.

40. Dias BR, Piovesana AMSG, Montenegro MA, Guerreiro MM. Desenvolvimento neuropsicomotor de lactentes filhos de mães que apresentaram hipertensão arterial na gestação. Arq. Neuro-Psiquiatr. 2005;63(3):632-666.

41. Kassar SB, Gurgel RQ, Albuquerque MFM, Barbieri MA, Lima MC. Peso ao nascer de recém-nascidos de mães adolescentes comparados com o de puérperas adultas jovens. Rev. Bras. Saude Mater. Infant. 2005; 5(3): 293-299.

42. Brock RS, Falcão MC. Avaliação nutricional do recém-nascido: limitações dos métodos atuais e novas perspectivas. Rev Paul Pediatr. 2008; 26 (1): 70-76.

Recebido em: 28/jul./2010

Modificado em 30/set./2010 Aceito em 10/fev./2011 